\title{
La modélisation des «marées vertes » littorales et ses applications
}

\author{
Alain Menesguen \\ IFREMER \\ Centre de Brest, BP 70, 29280 Plouzané
}

\section{Introduction}

La prolifération massive, au printemps et en été, d'algues vertes des genres Ulva et Enteromorpha est un phénomène observé depuis fort longtemps en de nombreux points du globe, ce qui laisse à penser qu'il s'agit au départ d'un phénomène écologique naturel. Mais dès le début du siècle, un lien net entre l'amplification du phénomène et l'augmentation locale des apports terrigènes de sels nutritifs azotés et phosphorés a été mis en évidence : LETTS et RICHARDS (1911), SAWYER (1965), STEFFEnSEN (1976), Soulsby et al. (1985). Les lagunes, semi-fermées, constituent naturellement les biotopes d'élection de ce genre de phénomènes d'eutrophisation : le lac de Tunis et surtout la lagune de Venise fournissent à cet égard les exemples les plus frappants de "marée verte ", avec une biomasse mesurée d'environ 550000 tonnes de poids frais en juin 1987 dans la lagune de Venise (SFriso et al., 1989). Le cas de la France est par contre très différent, puisque les neuf dixièmes des tonnages d'ulves ramassés sur les côtes françaises le sont sur les côtes de Manche-Atlantique, sur des estrans sableux largement ouverts vers le large et soumis à un important marnage (BRAULT, 1987). Qui plus est, la cartographie comparée des échouages d'ulves et des flux absolus de sels nutritifs apportés par les rivières ne révèle aucune corrélation apparente (PIRIOU, 1986), ce qui ajoute encore au caractère paradoxal de nos "marées vertes" françaises. En fait, la faiblesse explicative d'arguments globaux ou d'ordre qualitatif laisse à penser que ce sont les caractéristiques dynamiques fines des processus hydrodynamiques et biologiques qui conditionnent finalement le développement de "marées vertes" littorales. La modélisation numérique apparaît dès lors comme la technique la plus appropriée pour prendre en compte simultanément ces divers mécanismes et concevoir un outil à capacité prognostique.

\section{Description du modèle numérique}

Le modèle de base consiste en une représentation du système "ulves-sels nutritifs" au moyen de 5 variables d'état, dont l'évolution est régie par un système différentiel ordinaire non linéaire, appelé « sous-modèle biologique ». Ce dernier est soumis à l'action de deux variables forçantes climatiques : la température de la mer et le flux solaire incident dans la bande $400-700 \mathrm{~nm}$, utilisable par la photosynthèse.

Par ailleurs, ce système biologique est naturellement plongé dans un environnement physique aquatique, essentiellement caractérisé par des courants d'advection et de la dispersion. La représentation discrétisée de cet environnement hydrodynamique constitue le "sousmodèle physique ".

L'évolution temporelle du modèle écologique global résultant du couplage de ces deux sous-modèles est obtenue par une procédure d'intégration de RungeKutta d'ordre 4 à pas variable.

\section{Modelling Ulva coastal mass blooms with applications}

Excessive proliferation of Ulva sp. (Chlorophyta) during spring and summer was responsible for increasing injuries to recreational use of several beaches of Britanny (France) during the last decade. These worldwide mass blooms are well known in eutrophicated lagoons (Venice, Tunis...), but look paradoxical along open beaches with large tidal range and weak terrestrial nutrients inputs. Numerical modelling of the coupled physical and biological aspects clearly explains the Ulva mass blooms by the dynamical trapping of water masses in bays where tidal residual drift vanishes, and shows the main responsability of the increase of nitrogen inputs (of agricultural sources) in triggering the recent magnification of a natural phenomenon. 
Tableau I. Equations du sous-modèle biologique.

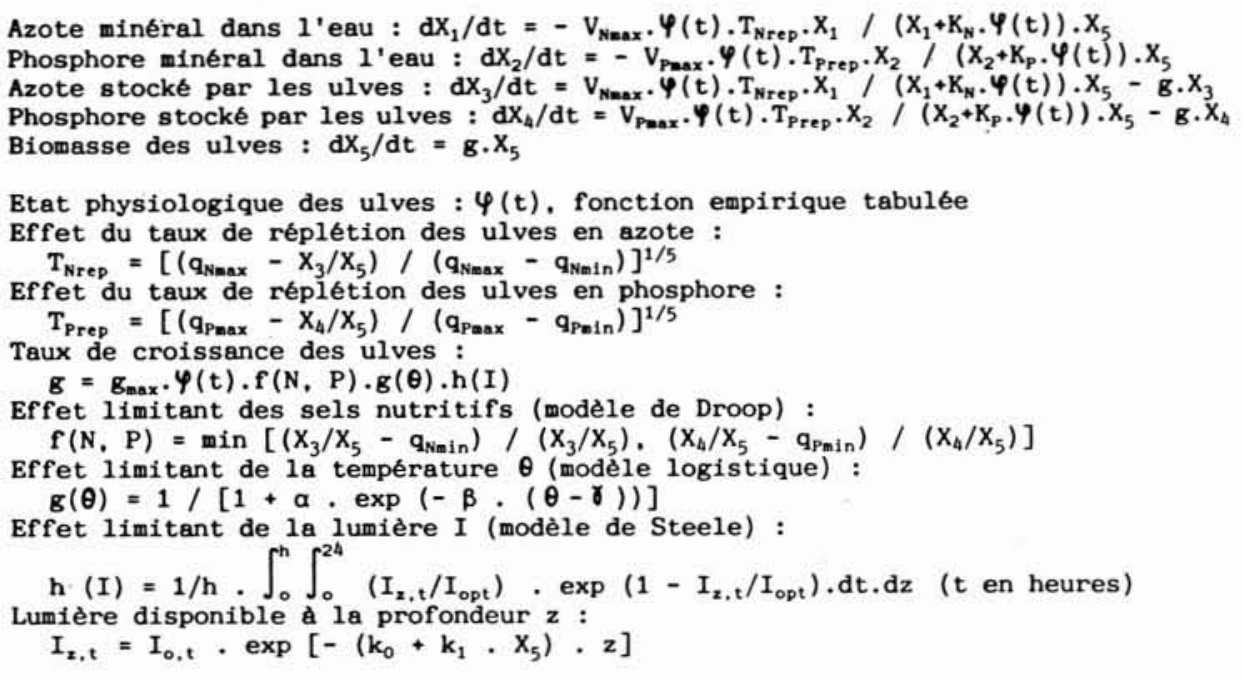

Tableau II. Paramètres du sous-modèle biologique.

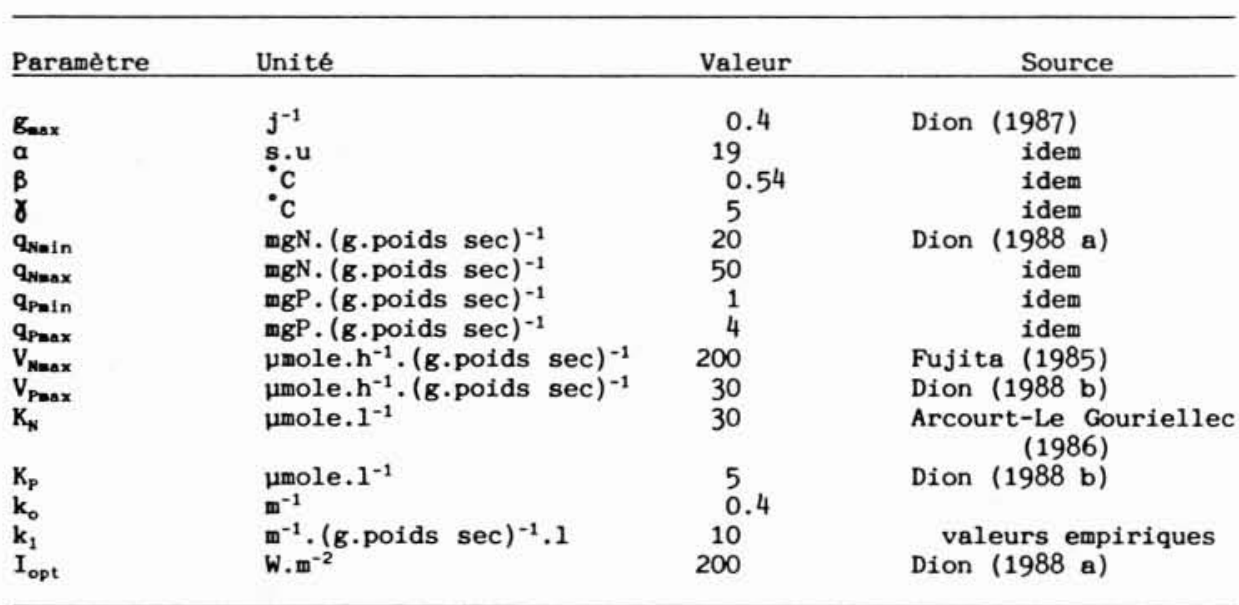

\subsection{Le sous-modèle biologique}

L'absence de prédateur notable des ulves croissant en pleine eau sur les plages fait que la biomasse d'ulves est essentiellement contrôlée par les compartiments amont des cycles naturels, c'est-à-dire ici l'azote et le phosphore sous forme minérale. Par ailleurs, de très nombreux travaux de physiologie végétale ont montré que le phytoplancton, et plus encore les macroalgues, sont capables d'un stockage temporaire de sels minéraux, ce qui déphase et amortit partiellement les variations du taux de croissance algal par rapport aux variations des sels nutritifs dans l'eau ambiante. Par analogie avec le modèle phytoplanctonique de DroOP (1970), on utilise donc les cinq variables d'état suivantes :
$X_{1}$ : azote minéral total dans l'eau

$X_{2}$ : phosphore minéral total dans l'eau

$X_{3}$ : azote total stocké dans les ulves

$X_{4}$ : phosphore total stocké dans les ulves $X_{5}$ : biomasse des ulves.

Le taux de croissance des ulves dépend non pas des concentrations dans l'eau $\left(X_{1}\right.$ et $\left.X_{2}\right)$, mais des quotas intracellulaires en azote et phosphore $\left(X_{3} / X_{5}\right.$ et $\left.X_{4} / X_{5}\right)$. La croissance estivale s'autolimite en raison de l'autoombrage créé par les fortes biomasses (Lapointe et Tenore, 1981). Le système différentiel est détaillé dans le tableau $I$ et les valeurs des paramètres sont fournies par le tableau II. La figure 1 fournit une représentation schématique du sous-modèle biologique. 


\subsection{Le sous-modèle physique}

La nécessité de pouvoir étudier la réponse théorique du système biologique sur des durées de plusieurs années ainsi que dans des cadres hydrodynamiques très variés conduit à utiliser un sous-modèle physique très rustique, appelé « modèle en boîtes ". Chaque boîte géographique peut recevoir des rejets, subir une prise d'eau, avoir un échange dispersif avec des sources extérieures et enfin avoir des échanges advectifs et/ou dispersifs avec les autres boîtes du système. La part hydrodynamique de l'évolution temporelle du $i$-ième constituant pélagique dans la $j$-ième boîte est donnée par l'équation suivante:

$$
\begin{aligned}
\mathrm{d} X_{i j / \mathrm{d} t}=\sum_{k=1}^{R_{j}} r_{j k} & \cdot X_{i k}-p_{j} \cdot X_{i j}+\sum_{k=1}^{N} \lambda \cdot A_{j k} \cdot X_{i k} \\
& +\sum_{k=1}^{N} \mu \cdot A_{j k} \cdot X_{i j}-\sum_{k=1}^{N} D_{j k} \cdot\left(X_{i j}-X_{i k}\right) \\
& -\sum_{k=1}^{S_{j}} E_{j k} \cdot\left(X_{i j}-X_{i k}\right)
\end{aligned}
$$

$r_{j k}=$ débit du $k$-ième rejet dans la boîte $j\left(R_{j}\right.$ rejets dans cette boîte $j$ ),

$p_{j}=$ débit de la prise d'eau dans la boîte $j$,

$A_{j k}=$ débit advectif de la boîte $k$ vers la boîte $j$

$$
\begin{array}{lll}
\lambda=1 & \mu=0 & \text { si } A_{j k}>0 \\
\lambda=0 & \mu=1 & \text { si } A_{j k}<0
\end{array}
$$

$D_{j k}=$ débit dispersif échangé entre les boîtes $j$ et $k$,

$E_{j k}=$ débit dispersif échangé entre la boîte $j$ et la $k$ ième source dispersive en contact avec la boîte $j$ $\left(S_{j}\right.$ sources en contact avec cette boîte $j$ ).

\section{Application du modèle écologique global}

\subsection{Choix et étude hydrodynamique du site}

Le site breton du fond de baie de St-Brieuc a été choisi du fait de son importante marée verte (la deuxième de France) et de l'existence d'un modèle hydrodynamique de cette zone (LeHAY, 1988). Un modèle détaillé du fond de la baie, utilisant des mailles de $250 \mathrm{~m}$ de côté, a permis de déterminer, à partir de la synthèse de trajectoires lagrangiennes débutant à diverses heures de la marée et s'achevant 12 h 25 min plus tard, une carte de courants résiduels lagrangiens en l'absence de vent (MENESGUEN et SALOMON, 1988). Représenté sur la figure 2, ce champ résiduel montre une nette dissymétrie entre la moitié ouest (à dérive plus ou moins forte) et la moitié est, où il existe une vaste zone à circulation résiduelle pratiquement nulle. Sur cette même figure 2, la position des accumulations d'algues déduite de photographies aériennes de juin 1986 montre clairement une bonne correspondance avec la zone à circulation résiduelle nulle. Ceci semble donc apporter une explication au développement paradoxal d'ulves sur estran largement ouvert vers le large en présence d'un fort marnage: les ulves peuvent être piégées dans des masses d'eau oscillant sur l'estran sans dérive latérale notable. Pour les besoins du modèle

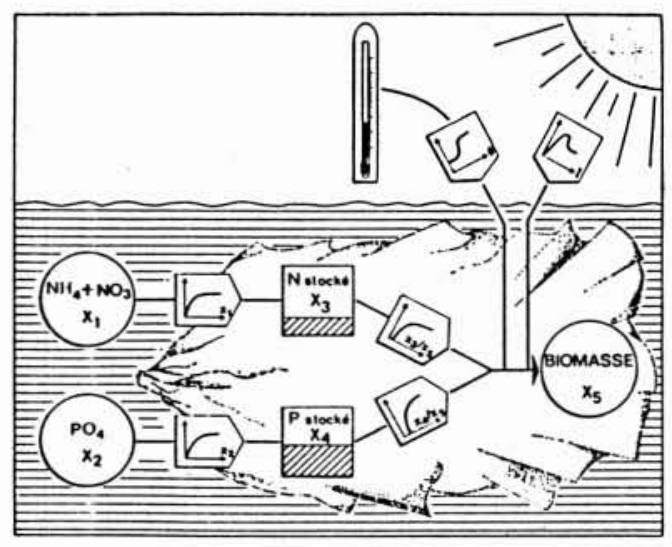

1. Schèma du sous-modèle biologique.

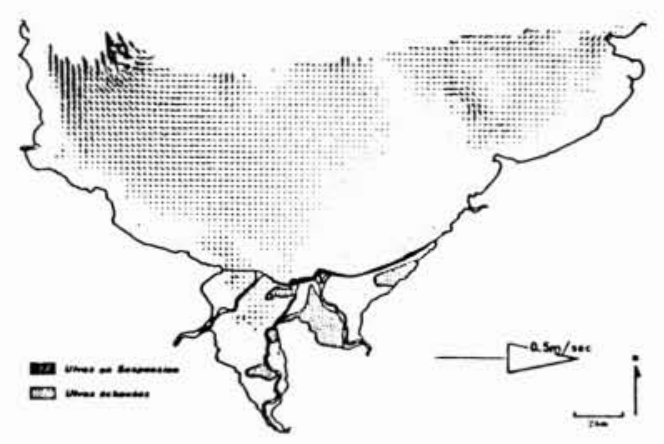

2. Baie de St-Brieuc : circulation résiduelle de marée et répartition des ulves à marée basse (juin 1986).

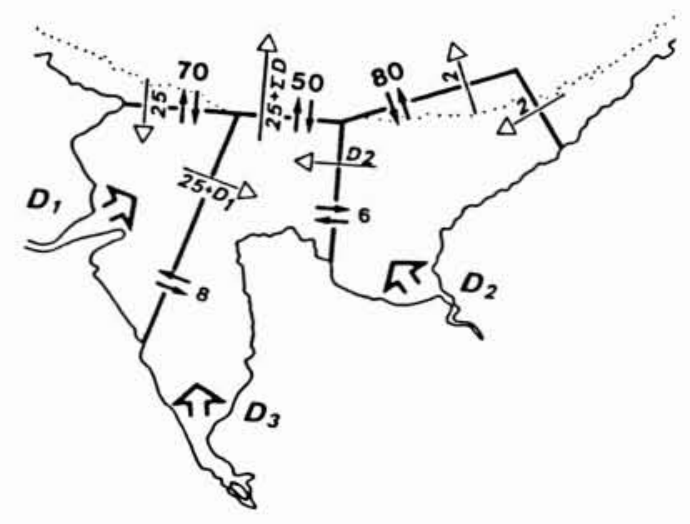

3. Schéma du sous-modèle hydrodynamique.

écologique, le modèle hydrodynamique a ensuite été réduit au système de 3 boîtes représenté sur la figure 3 ; les flux advectifs sont calculés d'après le champ résiduel précédent et les flux dispersifs, représentant le brassage par la marée, ont été estimés de façon à obtenir une salinité moyenne de $33,5 \%$ environ dans les 3 boîtes, de $3 \mathrm{~m}$ de profondeur. 


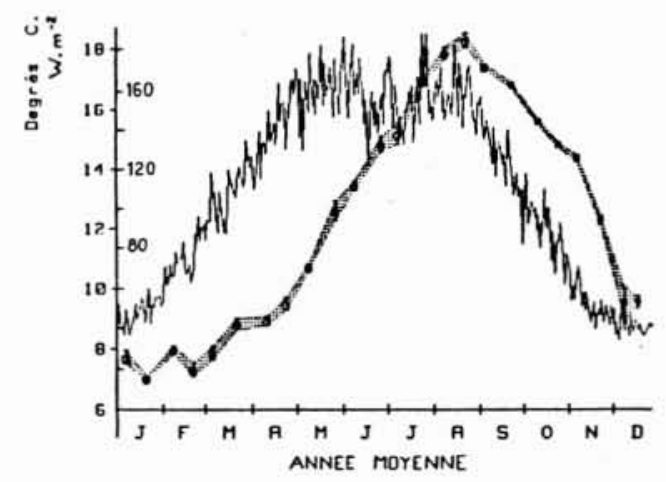

4. Température et éclairement en baie de St-Brieuc.

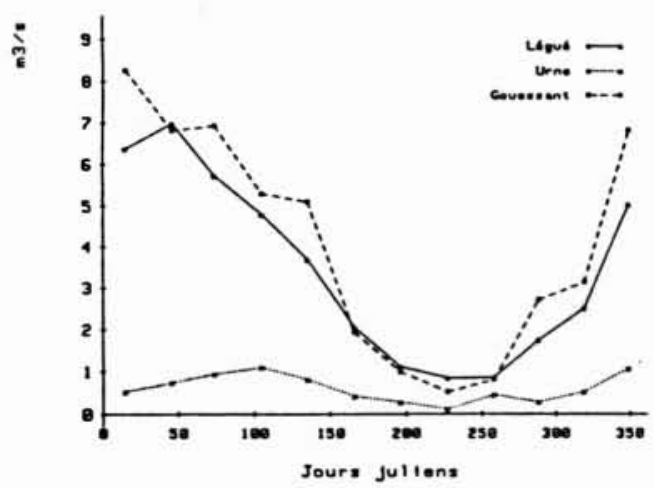

5. Débits des rivières concernées.

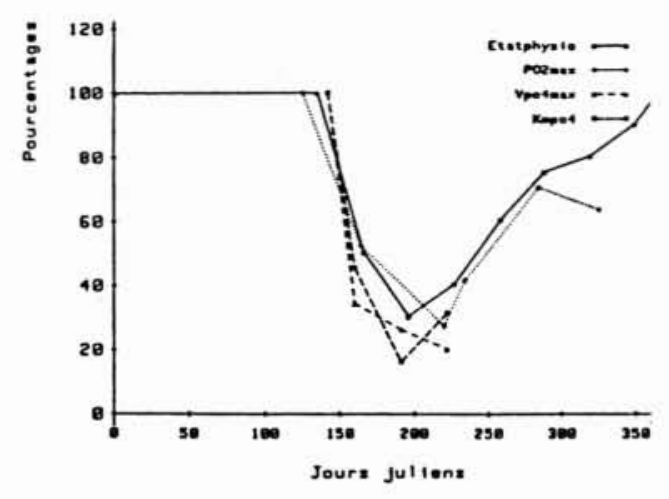

6. Etat physiologique de lulve.

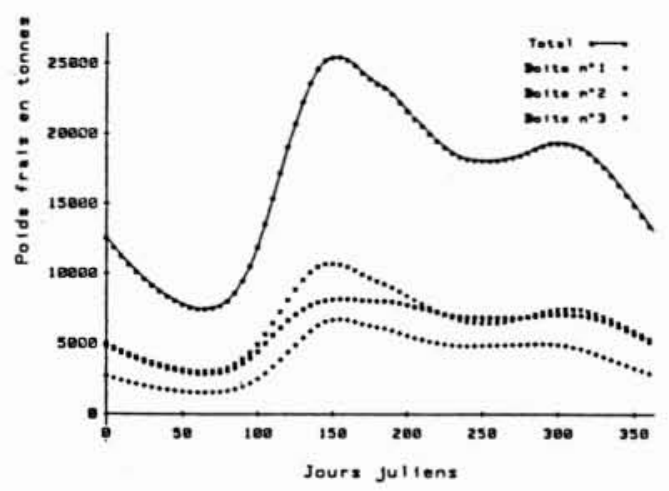

7. Evolution saisonnière des biomasses d'ulves.

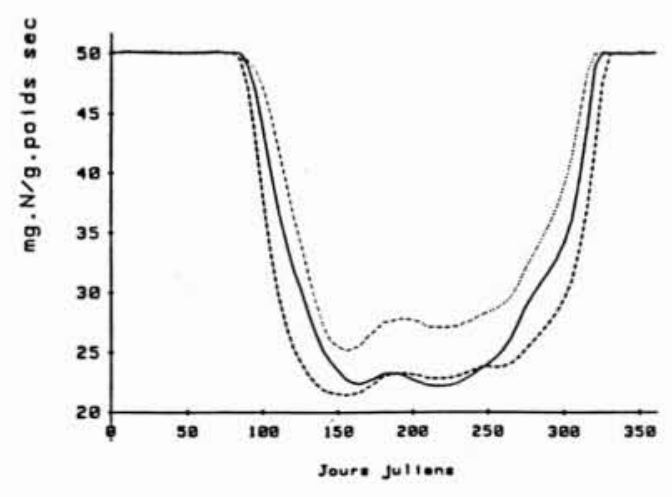

8. Evolution saisonnière du quota d'azote.

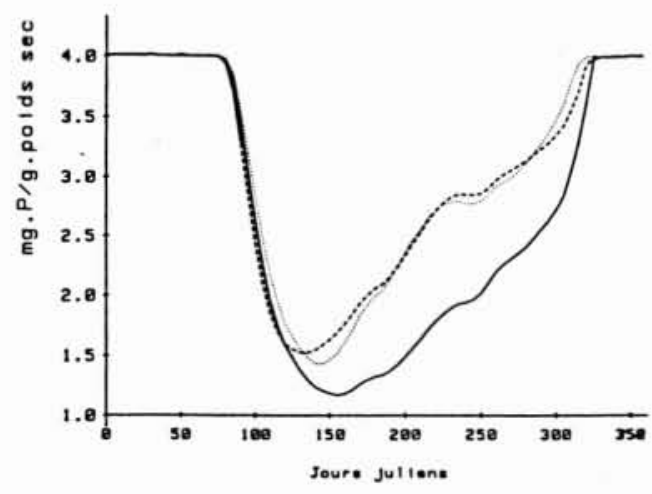

9. Evolution saisonnière du quota de phosphore.

\subsection{Simulation de la situation actuelle moyenne}

En l'absence d'une base de données synoptiques complète pour une année précise, on considérera une " année moyenne ", dont les caractéristiques sont obtenues par compilation des données existantes. La figure 4 présente l'évolution moyenne de la température aux stations RNO en baie de St-Brieuc, ainsi que celle de l'énergie lumineuse journalière moyenne disponible pour la photosynthèse, déduite des données d'insolation à Bréhat grâce à la procédure préconisée par Brock
(1981). La figure 5 présente la variation saisonnière du débit des trois rivières se jetant respectivement dans les trois boîtes du sous-modèle hydrodynamique. Le tableau III fournit les relations empiriques concentrations-débits pour ces trois rivières (MERCERON et al., 1981). La figure 6 donne enfin la variation de l'état physiologique de l'ulve déduite du cycle saisonnier mesuré pour divers paramètres physiologiques : intensité maximale de la photosynthèse (DION, 1988b), vitesse maximale et constante de Michaelis de l'absorption du phosphore (DION, 1988a). 
Tableau III. Concentrations des rivières en sels nutritifs (micromoles. $\mathrm{I}^{-1}$ ) en fonction de leur débit $\left(\mathrm{m}^{3} \cdot \mathrm{s}^{-1}\right)$.

\begin{tabular}{llll}
\hline & $\begin{array}{c}\text { Légué } \\
\text { (Boite 1) }\end{array}$ & $\begin{array}{c}\text { Urne } \\
\text { (Boite 3) }\end{array}$ & $\begin{array}{c}\text { Gouessant } \\
\text { (Boite 2) }\end{array}$ \\
\hline$\left[\mathrm{NH}_{4}\right]$ & $500 / \mathrm{D}_{1}$ & $4.4^{*} \mathrm{D}_{3}$ & $4.4^{*} \mathrm{D}_{2}$ \\
{$\left[\mathrm{NO}_{3}\right]$} & 320 & 320 & 320 \\
{$\left[\mathrm{PO}_{4}\right]$} & $30 / \mathrm{D}_{1}$ & $17 / \mathrm{D}_{3}$ & 6.5 \\
\hline
\end{tabular}

La simulation a été poursuivie jusqu'à obtention d'une évolution périodique du système, de période un an. Le terme de disparition de la biomasse a été considéré comme essentiellement contrôlé par les mouvements advectifs et dispersifs vers l'extérieur du système, le déplacement des ulves étant arbitrairement considéré comme vingt fois plus lent que celui des masses d'eau. La figure 7 donne l'évolution des biomasses, par boîte et globale, tandis que les figures 8 et 9 donnent l'évolution des quotas en azote et phosphore des algues. La biomasse maximale est bien obtenue dans la boîte 2, la plus à l'est, et la biomasse totale maximale évolue de façon comparable à ce qui a été estimé en 1986 (année oủ le ramassage fut tardif).

\subsection{Simulation de l'effet de réductions des apports}

La figure 10 montre les biomasses totales maximales attendues pour divers taux d'abattement des apports azotés et/ou phosphorés effectués simultanément sur les trois rivières. On constate qu'à taux d'abattement égal,

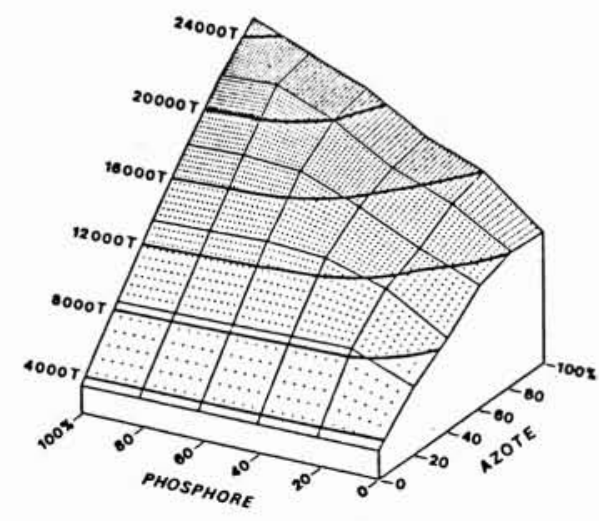

10. Biomasses totales maximales selon les apports.

une réduction des seuls apports d'azote s'avère plus efficace qu'une réduction des seuls apports de phosphore. La faible biomasse obtenue en supprimant totalement les seuls apports terrigènes d'azote ( 3500 tonnes) est à comparer avec celle obtenue en supprimant totalement les seuls apports terrigènes de phosphore (12000 tonnes): ceci semble indiquer que c'est l'augmentation récente des apports terrigènes d'azote qui a amplifié le phénomène de marée verte, et non l'augmentation des apports phosphorés. A l'appui de cette idée on remarquera qu'en présence de faibles apports azotés par les rivières, la biomasse produite devient quasiment insensible à l'importance des apports phosphorés.

\section{Discussion et conclusion}

Ce modèle numérique de "marée verte " s'est attaché à répondre à deux questions. Pour la première, qui concerne le mécanisme physique de confinement des algues sur des estrans macrotidaux largement ouverts sur le large, il montre que la topographie de certains fonds de baie peut conduire quasiment à l'annulation locale de la dérive résiduelle et piéger ainsi des masses d'eau. Ceci explique par ailleurs que l'intensité de l'eutrophisation à " marée verte" ne soit pas corrélée à la valeur absolue des apports terrigènes de sels nutritifs : un fort apport en zone à forte circulation résiduelle sera moins efficace qu'un faible apport en zone à résiduelle nulle. La seconde question concerne le rôle respectif de l'azote et du phosphore dans le contrôle des marées vertes. Le modèle penche en faveur de l'azote, ce qui s'accorde avec le caractère nitrophile des ulves et entéromorphes mentionné par plusieurs auteurs (LETTS et RICHARD (1911), Guist et Humm (1976), KAUTSKy (1982), CHAN et al. (1982), Fujita (1985)). A l'appui du rôle limitant de l'azote, on peut d'ailleurs citer les mesures bi-hebdomadaires de la composition des ulves en baie de StBrieuc obtenues en 1988: alors que le contenu azoté a montré un cycle habituel de déplétion au printemps et en été, le contenu phosphoré est resté maximal toute l'année (DION, 1988a). Enfin, il est prouvé de façon irréfutable que dans la lagune de Venise, la biomasse des ulves est contrôlée par les apports d'azote, le phosphore étant largement excédentaire (SFriso et al., 1989). Ceci n'exclut pas le fait qu'une déphosphatation très poussée, voire totale, des rejets ne puisse atténuer l'ampleur des " marées vertes " bretonnes. Mais il semble plus efficace (sinon plus facile!) de diminuer sensiblement les apports azotés, essentiellement d'origine agricole. 


\section{Bibliographie}

Arcourt-Le Gouriellec C. (1986). - Prise des ions $\mathrm{NH}_{4}^{+}$et $\mathrm{NO}_{3}^{-}$par la Chlorophycée Ulva lactuca L. aux stades protothalle et adulte : influence de l'éclairement incident. Rapport de DEA, Université de Nantes, 40 p.

Brault D. (1987), - Les marées vertes. Communication présentée au Colloque "Eutrophisation". Agence de Bassin Loire-Bretagne, 25-26 novembre 1987. Orléans.

BRock T. D. (1981). - Calculating solar radiation for ecological studies. Ecol. Model. 14, 1-19.

Chan K. Y., Wong P. K. \& NG S. L. (1982). - Growth of Enteromorpha linza in sewage effluent and sewage effluent-seawater mixtures. Hydrobiologia 97, 9-13.

Dion P. (1987). - Etude préliminaire à la déphosphatation en baie de St-Brieuc. Rapport CEVA/Pleubian pour le Conseil Général des Côtes d'Armor, 14 p.

Dion P. (1988a). - Etude de certains aspects écophysiologiques des marées vertes. Rapport de contrat CEVA/Pleubian pour l'IFREMER, $37 \mathrm{p}$.

Dıon P. (1988b). - Evaluation du rôle potentiel du phosphore dans la prolifération des ulves en baie de St-Brieuc. Rapport de synthèse IFREMER-CEVA/Pleubian pour le Conseil Général des Côtes d'Armor, 6 p. +8 figures.

Droop M. R. (1970). - Vitamin $B_{12}$ and marine ecology. V. Continuous culture as an approach to nutritional kinetics. Helgol. wiss. Meeresunters. 20, 629-636.

FuJITA R. M. (1985). - The role of nitrogen status in regulating transient ammonium uptake and nitrogen storage by macroalgae. J. exp. mar. Biol. Ecol, 92, 283-301.

Guist G. G. \& Нuмм H. J. (1976). - Effects of sewage effluents on growth of Ulva lactuca. Fla Sci. 4, 267-271.

KAUTSKY L. (1982). - Primary production and uptake kinetics of ammonium and phosphate by Enteromorpha compressa in an ammonium sulphate industry outlet area. Aquat. Bot. 12, 23-40.

LAPOINTE B. E. \& Tenore K. R. (1981). - Experimental outdoor studies with Ulva fasciata Delile. I. Interaction of light and nitrogen on nutrient uptake, growth and biochemical composition. J. exp. mar. Biol. Ecol. 53 , 135-152.

LEHAY D. (1989). - Etude de l'hydrologie et de l'hydrodynamique de la baie de St-Brieuc. Approche du rôle de l'hydrodynamisme sur la coquille St-Jacques. Thèse de Doctorat, Univ. Bretagne Occidentale, $338 \mathrm{p}$.

LETTS E. A. \& RICHARDS E. H. (1911). - Report on green seaweeds and especially Ulva latissima in relation to the pollution of the waters in which they occur. Royal Commisssion on Sewage Disposal. 7th Report, HMSO, London. Appendix III, Section II.

Menesguen A. \& SAlomon J. C. (1988). - Eutrophication modelling as a tool for fighting against Ulva coastal mass blooms. In : Computer Modelling in Ocean Engineering, Schrefler B. A. \& O. C. Zienkiewicz, eds. Proc. Intern. Conf, held in Venice, 19-23 septembre 1988.

Merceron M., Manaud F., Guillaud J. F. \& Monbet Y. (1981). - Extension du port du Légué (St-Brieuc). Etude d'impact sur l'environnement marin. Rapport CNEXO/COB/ELGMM pour la Chambre de Commerce et d'Industrie des Côtes d'Armor, $115 \mathrm{p}$.

PIRIOU J. Y. (1986). - Les marées vertes sur le littoral breton. Bilan 1985. Rapport IFREMER/DERO-86.29-EL.

SAWYER C. (1985). - The sea-lettuce problem in Boston Harbor. J. Wat. Poll. Control Federation, 37, 1122-1133.

Sfriso A., PAvoni B. \& Marcomini A. (1989). - Macroalgae and phytoplankton standing crops in the central Venice Lagoon : primary production and nutrient balance. The Science of the Total Environment, 80, 139-159.

Soulsby P. G., Lowthion D., Houston M. \& Montgomery H. A. C. (1985). - The role of sewage effluent in the accumulation of macroalgal mats on intertidal mudflats in two basins in Southern England. Neth. J. Sea Res. 19 (3/4), 257-263.

STEFFENSEN D. A. (1976). - The effect of nutrient enrichment and temperature on the growth in culture of Ulva lactuca L. Aquat. Bot., 2, 337-351. 